\title{
A clinical approach to the aetiology of uveitis in Bantu adults
}

\author{
JEFFREY FREEDMAN \\ From the University of Witwatersrand, St Yohn's Eye Hospital Baragwanath, Fohannesburg, South Africa, \\ and State University of New York, USA
}

Duke-Elder (1966) stated that, although numerous authors have published figures showing the incidence of different aetiological factors in uveitis, it is still difficult to obtain figures of an overall distribution because many papers deal with only one type of uveitis. Although a few clinical pictures can be recognized with some certainty, the aetiology of most cases of uveitis have persistently proved elusive. The results obtained after investigations into the aetiology of uveitis in adult Bantus in South Africa during a 2-year period are presented.

\section{Clinical study}

During a 2-year-period 3 II adult Bantus were seen; their sexes and ages are shown in Table I. The investigations of possible aetiological factors were divided into two main groups. In the first group all patients were examined by a predetermined set of laboratory and clinical studies. These comprised:

I. Radiological examination of chest, teeth, sinuses, and lumbo-sacral joints.

2. Blood tests including a full blood count, erythrocyte sedimentation rate (ESR), antistreptolysin titre, mucoprotein levels, blood sugar levels, gonococcal complement-fixation test, toxoplasma dye and complement-fixation tests, Rose latex test for arthritis, serum protein electrophoresis, histoplasma complement-fixation test, and Wassermann, Treponema pallidum immobilization, and fluorescein antibody tests for syphilis. Stools and urine were examined for parasites and micro-organisms, and there were skin tests for tuberculosis.

A second group of investigations based on the identification of specific clinical entities was also carried out. Here a presumptive aetiological diagnosis was made on clinical examination of the patients and confirmed by the appropriate special investigations where these existed.

The specific clinical entities seen in this study included: tuberculosis, sarcoidosis, sympathetic ophthalmitis, leprosy, syphilis, Vogt-Koyanagi-Harada syn-

Address for reprints: J. Freedman, MD, State University of New York, Downstate Medical Center, Department of Surgery, Division of Ophthalmology, 450 Clarkson Avenue, Brooklyn, New York 11203 , USA drome, intraocular parasites, toxoplasmosis, and presumed histoplasmosis syndrome.

\section{Group I (I 59 patients)}

In these patients there was no obvious clinical cause for the uveitis. Results of special investigations were as follows:

I. The full blood count results were non-contributory as regards significant abnormalities.

2. Altogether 27 patients had a Westergren ESR of $23 \mathrm{~mm} / \mathrm{rst}$ hour or greater (Table II). The highes 1 ESR recorded was 69 . Of the 159 patients 16 per cent had a raised ESR.

3. Gamma globulins were raised in I I patients (8 pe1 cent).

4. The Rose latex test for rheumatoid arthritis was positive in three patients none of whom had any clinical evidence of rheumatic disease.

5. The gonococcal complement-fixation test was positive in five patients none of whom had a urethra discharge concurrent with the uveitis.

6. The Wassermann reaction for syphilis was positivi in 20 patients.

7. The toxoplasmosis dye test was positive in $\mathrm{r}]$ patients.

8. The histoplasmosis complement-fixation test wa: positive in two patients, one of whom had fundu: lesions suggestive of the disease.

9. Urine analysis and stool investigation did not revea any parasites.

ro. Radiological investigations revealed three patient with active sinusitis.

Table I Age and sex distribution of Bantus with uveitis

\begin{tabular}{|c|c|c|}
\hline Age (years) & Males & Females \\
\hline $0-14$ & 14 & 27 \\
\hline $15-24$ & 35 & 20 \\
\hline $25-44$ & 88 & 80 \\
\hline $\begin{array}{l}45-64 \\
65+\end{array}$ & $\begin{array}{r}44 \\
4\end{array}$ & $\begin{array}{r}37 \\
6\end{array}$ \\
\hline Total & 185 & 170 \\
\hline
\end{tabular}


Table II Number and percentage of uveitis patients with positive results for routine serological and radiological investigations

\begin{tabular}{|c|c|c|}
\hline Test & Total & Per cent \\
\hline$X$-ray sinus & 3 & 2 \\
\hline ESR & 27 & I6 \\
\hline Mucoprotein & I3 & 9 \\
\hline Alpha globulin & I I & 8 \\
\hline Antistreptolysin titre & 9 & $5 \cdot 5$ \\
\hline Rose latex & 3 & 2 \\
\hline $\begin{array}{l}\text { Gonococcal complement- } \\
\text { fixation } \\
\text { Venereal Disease Reference }\end{array}$ & 5 & $3 \cdot 2$ \\
\hline Laboratory & 20 & 12 \\
\hline $\begin{array}{l}\text { Toxoplasma dye and } \\
\text { complement-fixation } \\
\text { Histoplasma complement- } \\
\text { fixation }\end{array}$ & I I & 7 \\
\hline
\end{tabular}

I I. Nine patients had an elevated antistreptolysin titre. These findings are summarized in Table II.

\section{Group 2}

This dealt with the following clinical entities in uveitis:

\section{Vogt-Koyanagi-Harada syndrome}

Two cases of this syndrome were seen, in two women aged 32 and 36 years, but neither presented with the full-blown clinical syndrome. The first patient presented with severe bilateral anterior uveitis characterized by intense scarring with extensive posterior synechiae, kerato-uveitis, and cataract formation. Depigmentation of the skin around the eyes with poliosis was present. No deafness or central nervous system involvement was noted. Although some scarring in terms of posterior synechiae occurred in both eyes, the visual acuity remained 20/20 in both eyes. A third case which had features of Vogt-Koyanagi-Harada syndrome was diagnosed presumptively as sympathetic ophthalmitis. A history of a penetrating injury to the left eye was given. The injured eye was not sutured and was removed I month later. Two weeks after injury the right eye became painful and developed a severe anterior uveitis with a final visual acuity of hand movements at $\mathrm{I} \mathrm{m}$. One month after the injury depigmentation of the skin of the face, legs, and hands occurred. No other features suggestive of Vogt-Koyanagi-Harada syndrome such as deafness or central nervous system involvement was noted. The diagnosis might have been Vogt-KoyanagiHarada syndrome or sympathetic ophthalmitis, the sequence of events suggesting the latter, but confirmation was denied as no histology of the enucleated eye was available. This case illustrates the clinical similiarity between sympathetic ophthalmitis and Vogt-KoyanagiHarada syndrome. In all three cases described scarring of the eyes occurred, and in two out of the three, the final visual acuity was poor.

\section{Leprosy}

Four cases of leprosy were seen, two in men and two in women. The two men presented with the tuberculoid type, each having bilateral facial nerve palsies, exposure keratitis, and secondary uveitis; one also had a hypopyon ulcer. The two women both presented with mixed form of leprosy-lepromatous and tuberculous. They both had primary anterior uveitis without associated seventh nerve palsies. In these two cases, scarring of the anterior segment was severe.

\section{Tuberculosis}

Two men presenting with uveitis were diagnosed as having tuberculous uveitis. The first patient presented with bilateral anterior uveitis. The left eye had no perception of light and optic atrophy and the right eye had papilloedema with diffuse active chorio-retinitis. The chest $x$ ray revealed active tuberculosis. The uveitis responded well with a combination of steroid and antituberculosis therapy. The second patient was a 35 -yearold man who presented with bilateral anterior uveitis with a right-sided proptosis. Once again a chest $x$ ray revealed active tuberculosis. In both these cases the clinical entity of uveitis due to tuberculosis was diagnosed because of the association of uveitis with active tuberculosis and this was further substantiated in the first case by what looked like a possible tuberculous chorio-retinitis.

\section{Sarcoidosis}

Two proved cases of sarcoidosis were seen. The first, a woman aged 38 years, presented with bilateral anterior granulomatous uveitis. She developed diffuse posterior synechiae in both eyes, the fundus being normal. The second case, a man aged 30 years, presented with bilateral granulomatous uveitis with inferior vitreous opacities. After treatment with systemic and local steroids both patients had a final visual acuity of $20 / 20$ in both eyes. In addition both patients had positive Kveim tests. Diagnosis in the woman was further substantiated by positive histology after scalene node biopsy. She suffered recurrent attacks of uveitis over a I-year-period and the man had a single recurrence in a follow-up of $\mathbf{I}$ year.

\section{Syphilis}

The diagnosis of syphilitic uveitis was based on a clinical appearance suggestive of the disease, associated with positive serology. Spirochaetes were not detected in the aqueous tested and no cases having positive serology but without typical clinical features are included. Altogether four presumptive syphilitic uveitis cases were seen, two being in men and two in women. The first man aged 19 years presented with bilateral vitreous haemorrhages and bilateral anterior uveitis. A strongly positive Treponema pallidum immobilization (TPI) test was present and no blood dyscrasia was noted. Marked improvement of the vitreous haemorrhages occurred with antibiotic therapy and systemic steroids. The second man presented with bilateral anterior uveitis 
with a left-sided hyphaema and associated fundal haemorrhages; a strongly positive Wassermann test was present. The first woman presented with a large inferior ciliary staphyloma and a lens dislocated into the vitreous in the right eye. The vitreous contained many cells as did the anterior chamber. The left eye had an anterior and posterior uveitis with an exudative detachment. There was no history of trauma and the TPI test, but not the Venereal Disease Reference Laboratory (VDRL) test, was positive. Thus, longstanding syphilis was diagnosed. The second woman presented with bilateral diffuse uveitis with exudative lesions of the choroid, as well as old areas of chorioretinitis. Again the TPI test was positive and the VDRL test negative, suggesting long-standing syphilis.

\section{Parasitic infections}

One patient, a 45-year-old man, presented with a uveitis and associated parasitic infestation. The right eye had an anterior uveitis with cells in the vitreous as well. Within the vitreous a large, live cysticercus was found associated with a hole at the macula. The diagnosis was made by direct observation of the parasite; no ova were obtainable from the patient's stools.

\section{Ankylosing spondylitis}

Two patients with ankylosing spondylitis and uveitis were seen. The first, a 27-year-old man, presented with a left-sided nongranulomatous uveitis. Both patients had typical clinical and $x$ ray features of ankylosing spondylitis.

\section{Toxoplasmosis}

Only one case with a typical fundus lesion and positive serology for toxoplasmosis was seen.

\section{Septicaemic retinitis}

A 35-year-old woman who had undergone renal transplants developed a perinephric abscess. Associated with this she developed a septicaemic retinitis and associated anterior uveitis.

No cases of chronic cyclitis were seen in the adult patients.

\section{Discussion}

In the 159 patients examined fully by clinical and laboratory investigations the significance of the results of the nonspecific tests was as follows:

\section{ESR}

This was found to be positive in 16 per cent of patients tested. Norn (1969) found the ESR to be raised in 52 per cent of his cases of uveitis. Leira (1965) found less marked but analogous percentages. The raised ESR indicates general illness such as arthritis or severe forms of uveitis and recrudescences. It seems that, in the absence of arthritis, in cases studied in the Bantu, the raised ESR was due to a severe form of uveitis, which was confirmed clinically. In those cases in which the ESR was raised it was significantly high. It is said to be a bad prognostic sign if the ESR is very high (Norn, 1969) and this certainly seemed to be so in the Bantus; in most of those with a raised ESR, the attacks of uveitis had been severe with the subsequent detrimental sequelae. Thus the ESR may be of prognostic rather than of specific aetiological value.

\section{Serum electrophoresis}

In this study, where abnormalities in serum proteins existed ( 7 per cent of cases), the common abnormality was a rise in the gamma globulins. These findings agree with those of Haut and Campinchi (1973) but the percentages are much lower in the Bantu. A rise in alpha-globulins as noted by Sokolovsky (quoted by Dieckhues and Böke, 1963) and alpha-2-globulins (Aronson, I968) was not found in the Bantus. The abnormalities noted in the serum proteins are probably only an indication of the reaction of the individual to the uveitis as stated by Aronson (1968) and probably of no aetiological significance. No specific aetiological agent was noted in any case with raised gamma globulins in this study. The Wassermann reaction for syphilis was positive in 12 per cent of cases of uveitis on routine study. In none of these cases was any improvement noted with specific therapy nor was the uveitis typical of that caused by syphilis and the positive Wassermann reaction was probably a coincidental finding. This is again suggested by the fact that 20 per cent of the Bantu population have a positive Wassermann reaction. Therefore, the Wassermann test played an insignificant role in assisting to elucidate an aetiological factor in the patients undergoing routine investigations for uveitis.

The latex test for rheumatoid factor was positive in 2 per cent of the Bantu patients. However, none of these patients manifested any clinical evidence of arthritis and on routine investigation this was not a helpful diagnostic procedure.

Serology for gonorrhoea was positive in 3.2 per cent of the cases studied. This figure is similar to the percentage found by Norn (1969) in his series. However, the uveitis seen in the patients of this study were not typical of gonococcalinduced uveitis and no real improvement occurred with specific therapy. The positive gonococcal serology was not assessed to mean that the uveitis was due to gonococcal organisms. 
Routine investigation for toxoplasmosis was ested by two methods, the Sabin-Feldman dye est and the complement-fixation test. The positive esults were obtained in 7 per cent of patients on outine testing. However, in none of those was here a rising titre on re-testing, nor was the titre ligher than I:I28. Of the I I patients with positive ests for toxoplasmosis, six had anterior uveitis vith no associated fundus lesion, the uveitis probibly not being due to Toxoplasma gondii. The emaining five patients had fundus lesions only one of which resembled that due to toxoplasmosis. Dnce again for routine investigation, the toxoJlasma serological tests were of no help in the liagnosis of aetiological agents in cases of anterior Iveitis.

On routine investigation the histoplasma complenent-fixation test was positive in two patients, one of whom had a fundus lesion which was not typical of histoplasmosis and on repeat testing a positive istoplasma result was not obtainable. Histoplasnosis appears not to be a common cause of uveitis n Bantus of South Africa.

Thus in an overall general investigation of 159 idult patients with uveitis, the gleaning in terms of etiological agents was small.

\section{ZLINICAL ENTITIES}

Jf the two cases diagnosed as Vogt-Koyanagi yndrome, one patient presented with the uveitis irst and then the vitiligo, while the second presented vith uveitis and vitiligo simultaneously. Both these resentations are acceptable for the diagnosis of Togt-Koyanagi syndrome. In its classical form he uveitis precedes the vitiligo by 3 weeks to 3 honths. Both patients were in their twenties which $s$ the classically described age group. The presenation of diffuse bilateral uveitis is also typical of he disease, as is the poor visual result and scarring $s$ seen in one of the patients. No deafness or central ervous system involvement occurred in either atient but these manifestations occur in only o per cent of patients with the disease. It is not urprising that the condition was seen in two lantus as it obviously predominates in the Far ast, Latin America, and the Mediterranean basin, nd is therefore an affliction of the heavily pigrented races-yellow and black. The first descripion in the Bantu was that of Trope and Coetzee I96I). Bruno and McPherson (I949) described he condition in the North American Negro. irgical intervention, such as lens extraction, does ot always give a good result and the prognosis hould be guarded. The poor result obtained fter lens extraction in one of the Bantu patients ertainly followed this dictum. The close inter- relationship between sympathetic ophthalmitis and Vogt-Koyanagi-Harada syndrome has been shown clinically in the Bantu first by Trope and Coetzee (196r) and possibly in the case described in this study. This clinical association has been further substantiated on an immunological level by the experiments of Wong, Anderson, and O'Brien (197I), and Hammer (197I), wherein they demonstrated positive lymphocyte transformation in the two conditions suggesting an immunological basis for them. Thus typical forms of VogtKoyanagi-Harada syndrome do occur in the Bantus of South Africa.

The incidence of tuberculosis as a possible cause for uveitis was less than I per cent in this study. In the Indiana Survey (Schlaegel, 1968) the incidence was I per cent and Haut (1966) found it to be 1.8 per cent in his survey. For Cross (1960) tuberculosis represented 14.6 per cent of chronic or recurrent uveitis, while Remky (I960) listed Io per cent of cases of uveitis due to tuberculosis. There tends to be a balance between the severity of the tuberculosis in the lungs and the uvea. Woods (1973) rarely found active pulmonary tuberculosis in the course of uveal tuberculosis. This would have appeared to be the findings in the two Bantu patients described, in that, although many findings were diagnostic of tuberculosis no bacilli were found in the sputum during the manifestation of the uveitis. The diagnosis of tuberculous uveitis in these two cases remains presumptive as Koch's postulates were not fulfilled. However, the feature brought out by the findings in this study is the now accepted one of a low to nonexistent incidence of tuberculous uveitis, particularly in the Bantus.

The two cases of sarcoidosis seen in this study represent an incidence of less than I per cent. Sarcoid is said to be an aetiological factor in approximately 3 per cent of cases of uveitis (Woods, 1973). The incidence in the Bantu is unlike that in the American Negro who appears to be abnormally susceptible to the disease. Although sarcoid uveitis is rare in the Bantu, the nature of the uveitis when it does occur is typical of sarcoid uveitis in other racial groups. The incidence of sarcoid in the Bantu is similar to that in the Caucasoid group ( 0.5 per cent; Haut, 1966), and unlike the frequency of sarcoid uveitis as seen in the American Negro.

Presumptive syphilitic uveitis diagnosed in four patients represents an incidence of $\mathrm{I} \cdot 3$ per cent. Old statistics showed a 25 to 75 per cent incidence and Woods (1973) recognized 4 per cent of uveitis as being due to syphilis. The incidence in Baltimore, USA, as reported by Perkins (1968), was $15^{\circ 2}$ per cent. Haut (1966) found an incidence of $I \cdot I$ per cent and Witmer (r969) stated that syphilitic 
uveitis was rare. A number between I and 4 per cent probably corresponds to the incidence of syphilitic uveitis. Thus the incidence of possible syphilitic uveitis in the Bantu corresponds to the accepted average and is low when considered against the positive serology in the general Bantu population which is 20 to 25 per cent. The diagnosis in the four Bantu patients remains presumptive as no firm evidence such as locating the spirochaete from the eye was obtained. However, the clinical evidence presented by the four patients indicated that the uveitis was due to syphilis. A hyphaema may be one of the characteristic findings of secondary syphilis. The presence of exudative chorioretinitis is also highly suggestive of syphilis. Uveitis of late syphilis may present with a cataract and ciliary staphyloma probably due to a gumma. Finally, the Treponema pallidium immobilization test is negative in normal persons and permits the elimination of false positives. The reaction to the test appears later than other serological reactions and remains positive in a late stage of the disease, being little influenced by therapy. Its positivity in all four cases with the associated clinical uveitis is indicative of the presence of syphilitic uveitis. However, even with the presumptive I per cent incidence, syphilis does not seem to be a major aetiological factor in the causation of uveitis in the Bantus of South Africa.

Four cases of leprosy represent $\mathrm{I} \cdot 3$ per cent incidence of leprosy uveitis. The comparison of the incidence of uveitis due to leprosy in other surveys is difficult, as in most surveys the incidence of uveitis in patients with leprosy is not given (Ticho and Bensira, 1970). The two women with mixed leprosy presented with the more severe uveitis as would be expected with lepromatous, or direct involvement uveitis, as compared with the tuberculoid form as seen in the two men. In this study, uveitis was caused by leprosy more often than by sarcoidosis or tuberculosis and presented an easily identifiable clinical entity.

There were only two cases of ankylosing spondylitis associated with uveitis, an incidence of less than I per cent. Blagojevic, Parunovic, Nickolick, and Mladenovic (1970) found an incidence in their series of 140 cases of 5 to 20 per cent. Ankylosing spondylitis in association with uveitis is rare in the Bantu and the two cases associated with uveitis are unique.
Only one case with a typical fundus lesion anc positive serology for toxoplasmosis was seen. Thisrepresents an incidence of 0.3 per cent of uveitis due to toxoplasmosis in the Bantu studied. Var. Metre (1968) found an incidence of 14 per cent anc Chesterton and Perkins (1967) an incidence of 8 per cent of which 34 per cent were Negroeses from the West Indies or West Africa. Hogan $\vec{O}$ Kimura, and O'Connor (1964), in a study o: uveitis in 1584 patients, found 240 patients ( $1: \omega$ per cent) with suspected ocular toxoplasmosis few of these were Negroes. The explanation for? this observation was given. The study in Bantus? in which a very low incidence of toxoplasmic uveitis was seen, correlates well with Hogan':\$ observations in American Negroes. On routins testing, a positive serological test for toxoplasmosiwas obtained in 7 per cent of the Bantu patientso none of whom had features of ocular toxoplas? mosis. This finding of positive serology for toxo plasmosis correlates with the incidence of positive serology for the Bantu population as a whole which is ro per cent. From the findings of thi? study, the nonspecific testing for toxoplasmosio in all cases of uveitis would seem to be unhelpfus in elucidating an aetiological factor, and the searclo for clinical features of toxoplasmosis with subse $\frac{}{8}$ quent serological testing would seem to be mor尺 practical.

\section{Conclusions}

The gleanings in terms of an aetiological diagnosio․ obtained from nonspecific blanket testing of af cases of uveitis would seem to be poor in th 3 Bantus. Where positive aetiological factors wer obtained, a clinical diagnosis was more often thas not possible, the diagnosis being confirmed by tho appropriate special tests. It would seem ther? from the findings of this study, that routine ino vestigations of all cases of uveitis along presen lines, as advocated by Woods (1961), are usuallo fruitless, and that the identification of clinict entities, as suggested by Maumenee (1968), is $\underset{\mathrm{N}}{ }$ more logical approach in the investigation uveitis.

I should like to thank the Medical Superintendent $\stackrel{\mathscr{\infty}}{+}$ Baragwarath Hospital for allowing access to hospit:0 records.

\section{References}

ARONSON, S. B. (1968) In 'Clinical Methods in Uveitis', pp. 185-195. Mosby, St Louis

Blagojevic, M., PaRunovic, A., Nickolick, J., and mladenovic, v. (i970) Comm. An. 77'eme Congrès de la

Société française d'Ophtalmologie

BRUNo, M. G., and MCPHERSON, s. D. (1949) Amer. F. Ophthal., 32, 5 I3 


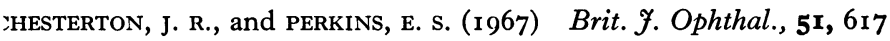

'Ross, A. G. (1960) Docum. ophthal. (Den Haag), 14, 223

jIECKHUES, B., and вöкE, w. (1963) $\quad$ v. Graefes Arch. Ophthal., 166, 249

JUKE-ELDER, STEWART (1966) In 'System of Ophthalmology, Diseases of the Uveal Tract', pp. 85-86. Kimpton,

London

IAMMER, H. (1971) Brit. F. Ophthal., 55, 850

iAUT, J. (1966) Arch. Ophtal. Paris, 26, 691

and CAMPINCHI, R. (1973) In 'Uveitis. Immunologic and Allergic Phenomena', by R. Campinchi,

J. P. Faure, E. Bloch Michel, and J. Haut, trans. B. Golden and M. M. Givioset. Thomas, Illinois

IOGAN, M. J., KIMURA, S. J., and o'CONNOR, R. G. (I964) Arch. Ophthal., 72, 592

.EIRA, H. (1965) Acta ophthal. (Kbh.), Suppl. 85, 201

IAUMENEE, A. E. (I968) In 'Clinical Methods in Uveitis', by S. B. Aronson, pp. 848-864. Mosby, St Louis JORN, M. S. (1969) Acta ophthal. (Kbh.), 47, 848

'ERKINS, E. S. (1968) In 'Clinical Methods in Uveitis', by S. B. Aronson, pp. 82-85. Mosby, St Louis ЕMKY, H. (г960) Bull. Soc. franc. Ophtal., 73, 64

ChlaEgel, T. F. (I 968) In 'Clinical Methods in Uveitis', by S. B. Aronson, pp. 82-85. Mosby, St Louis ICHO, U., and BENSIRA, I. (1970) Brit. F. Ophthal., 54, IO7

'ROPE, R. A., and COETZEe, D. C. (I96r) Med. Proc., 7, 7 I

'AN METre, JR., T. E. (I968) In 'Clinical Methods in Uveitis', by S. B. Aronson, p. I03. Mosby, St Louis

NITMER, R. (1969) Communication au Symposium sur les Uveites, Société Italienne d'Ophtalmologie, Messine vONG, v. G., ANDERSON, R., and o'brien, J. (I97I) Amer. F. Ophthal., 72, 960

vooDs, A. c. (I96I) In 'Endogenous Inflammation of the Uveal Tract'. Williams \& Wilkins, Baltimore (1973) Quoted by: E. Bloch Michel, In 'Uveitis. Immunologic and Allergic Phenomena', by

R. Campinchi, J. P. Faure, E. Bloch Michel, J. Haut. Thomas, Illinois 